\title{
Study of the shell effect on nuclear dissipation via neutron multiplicity measurement
}

\author{
Varinderjit Singh $^{1 *, a}$, B. R. Behera ${ }^{1}$, Jhilam Sadhukhan ${ }^{2, b}$, and Santanu Pal ${ }^{2}$ \\ ${ }^{1}$ Department of Physics, Panjab University, Chandigarh 160014, India. \\ ${ }^{2}$ Variable Energy Cyclotron Centre, 1/AF, Bidhan Nagar, Kolkata 700064, India.
}

\begin{abstract}
Experimental neutron multiplicity data has been fitted using the Kramers' fission width, in statistical model calculations with and without inclusion of shell corrections in nuclear level density, nuclear masses and fission barrier. It has been found that the magnitude of dissipation strength changes to a large extent with inclusion of shell corrections in different ingredients of the statistical model. These observations clearly demonstrate the importance of shell corrections in statistical model calculations.
\end{abstract}

\section{Introduction}

It is now well established that the Kramers' fission width is necessary to describe fusion-fission reaction dynamics. The experimentally obtained neutron multiplicity, charged particle multiplicity, GDR $\gamma$-rays multiplicity and ER cross-sections are found to be in excess than the values predicted by the standard statistical model using the Bohr-Wheeler fission width [1]. From the study of a large set of experimental data, it is found that nuclear dissipation plays a vital role in fission dynamics for nuclear temperature above $1 \mathrm{MeV}$ [2]. It is further observed that nuclear dissipation has an influence on various nuclear processes such as the decay of excited compound nuclei $(\mathrm{CN})$, survival probability of super heavy elements (SHE), deep inelastic scattering etc. Hence it is necessary to understand the magnitude and nature of nuclear dissipation. Also it is important to know how the nuclear dissipation depends on the excitation energy and the shell effects. The understanding of shell effects on nuclear dissipation is important for the synthesis of the theoretically predicted SHE. Attempts are continuing to synthesise the SHE using the cold fusion process, where the $\mathrm{CN}$ excitation energy may vary from 30 to $40 \mathrm{MeV}$. The shell effects can play an important role in this excitation energy range and therefore shell closure in $\mathrm{CN}$ can influence the survival probability of SHE.

With the motivation to investigate the effect of shell closure in $\mathrm{CN}$ on nuclear dissipation, a series of experiments were carried out to measure the neutron multiplicity using the National Array of Neutron Detectors (NAND) at Inter University Accelerator Centre (IUAC), New Delhi. Three

\footnotetext{
*Corresponding author: Mangat_phy@yahoo.co.in

${ }^{a}$ Present address: Centre for Physical and Mathematical Sciences, Central University of Punjab, Bathinda 151001, India.

${ }^{b}$ Present address: Department of Physics and Astronomy, University of Tennessee, Knoxville, Tennessee 37996, USA and Physics Division, Oak Ridge National Laboratory, Oak Ridge, Tennessee 37831, USA.
} 
different isotopes of $\operatorname{Fr}\left({ }^{213,215,217} \mathrm{Fr}\right)$ were populated using the ${ }^{19} \mathrm{~F}+{ }^{194,196,198} \mathrm{Pt}$ reactions [3]. Out of these three isotopes, ${ }^{213} \mathrm{Fr}\left(\mathrm{N}_{\mathrm{c}}=126\right)$ is a shell closed $\mathrm{CN}$ and the other two $\left({ }^{215} \mathrm{Fr}\right.$ and $\left.{ }^{217} \mathrm{Fr}\right)$ are away from shell closure with neutron numbers 128 and 130 respectively. The experimental neutron multiplicity was employed to extract the magnitude of nuclear dissipation. In the present investigation, we shall demonstrate the importance of shell correction in various input quantities to statistical model (nuclear masses and fission barrier in particular) to estimate the nuclear dissipation strength by fitting the experimental data.

\section{Statistical model calculations}

The statistical model used in the present study considers emission of light particles (neutrons, protons, $\alpha$-particles and $\gamma$-rays) and fission as the possible modes of decay of an excited $\mathrm{CN}$. The partial widths for light charged particles and GDR $\gamma$-rays are obtained using the Weisskopf formula [4]. The spin dependent fission barrier is calculated using the finite range liquid drop model (LDM) for nuclear potential [5] and the rigid rotor values for moment of inertia. The decay widths of various decay channels are sensitive to the density of levels (level density parameter) in the initial and final states. The level density parameter is taken from the work of Ignatyuk et al. [6], who proposed an expression for level density which includes the excitation energy dependent shell corrections. Both the Bohr-Wheeler fission width and the Kramers' fission width are used in the statistical model calculations. The Kramers' fission width is given as;

$$
\Gamma_{K}=\frac{\hbar \omega_{g}}{2 \pi} e^{-V_{b} / T}\left\{\sqrt{1+\left(\frac{\beta}{2 \omega_{s}}\right)^{2}}-\frac{\beta}{2 \omega_{s}}\right\}
$$

where $\omega_{g}$ and $\omega_{s}$ are the local frequencies of the harmonic oscillator potentials in the ground state and saddle state configurations respectively. Here $\beta$ (dissipation coefficient $\gamma=\beta / 2 \omega_{s}$ ) is the reduced dissipation coefficient which can be treated as a free parameter to fit the experimental data. Further details about the model can be found in Refs. [3, 7].

\section{Results and Discussion}

Statistical model analysis of neutron multiplicities for ${ }^{217} \mathrm{Fr},{ }^{215} \mathrm{Fr}$ and ${ }^{213} \mathrm{Fr}$ compound nuclei were performed in an earlier work [3] where the importance of including shell effects in nuclear level density and fission barrier was demonstrated. The neutron (and proton and alpha) binding energies were obtained from experimental nuclear masses in the above work. In the following, we shall briefly discuss how the shell effect on neutron binding energy and fission barrier affects the fitted values of the dissipation strength. To this end, we shall compare statistical model predictions obtained with the liquid drop model (LDM) nuclear mass to calculate the neutron binding energy with those obtained with the experimental nuclear masses. A detailed account of this work is given in Ref. [8].

We first compare the experimentally obtained neutron multiplicity for ${ }^{217} \mathrm{Fr},{ }^{215} \mathrm{Fr}$ and ${ }^{213} \mathrm{Fr}$ compound nuclei with the statistical model predictions using the Bohr-Wheeler fission width along with the LDM masses and no shell correction in level density and fission barrier. It is found that the statistical model with Bohr-Wheeler fission width underestimates the pre-scission neutron multiplicity $\left(\mathrm{M}_{\text {pre }}\right)$ when compared with the experimental data. Kramers' fission width is then employed to fit the experimental $\mathrm{M}_{\text {pre }}$ using the reduced dissipation coefficient $(\beta)$ as a free parameter. It is observed that an excitation energy dependent $\beta$ is required to fit the experimental neutron multiplicity as shown in Figure 1(a). This figure also shows that nearly the same dissipation strengths are required to fit prescission neutron multiplicities for all the isotopes of Fr under study.

We have next performed the statistical model calculations, where shell effects are included in the level density but not in the fission barrier. The LDM masses are used in this calculation to obtain the binding energies of the evaporated particles. It is again found that the Bohr-Wheeler fission width under predicts $\mathrm{M}_{\text {pre }}$ and an excitation energy dependent $\beta$ is required to fit the experimental values of $\mathrm{M}_{\text {pre }}$. Comparison of the fitted dissipation strengths (Figure 1(b)) shows that the dissipation strength is 
highest for ${ }^{217} \mathrm{Fr}$ and lowest for ${ }^{213} \mathrm{Fr}$. It is also noted that the dissipation strengths for ${ }^{217} \mathrm{Fr}$ and ${ }^{215} \mathrm{Fr}$ are quite close to each other whereas the same is smaller for ${ }^{213} \mathrm{Fr}$ compared to the other two isotopes. This observation is similar to the one made by Back et al. [9] which led them to conclude that the shell closure in $\mathrm{CN}$ results in lowering of the dissipation strength.

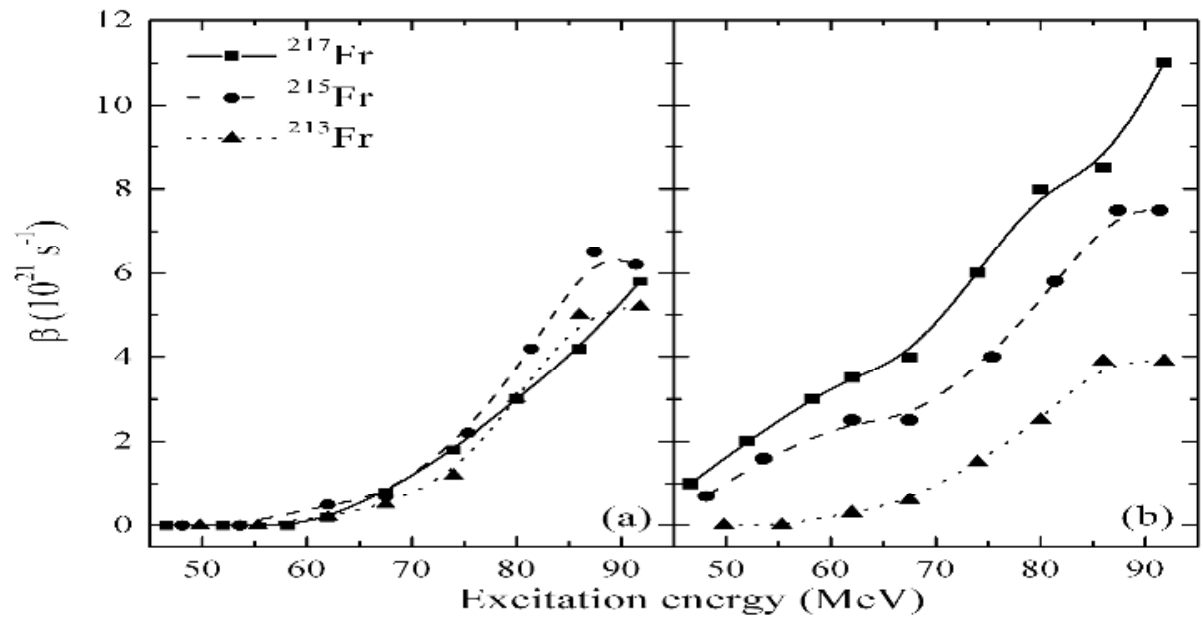

Figure 1: Comparison of the dissipation strength $(\beta)$ required to fit the experimental $M_{\text {pre }}$ for different isotopes of Fr using LDM masses and (a) without any shell correction in level density and fission barrier (b) with shell corrected level density and no shell correction in fission barrier.

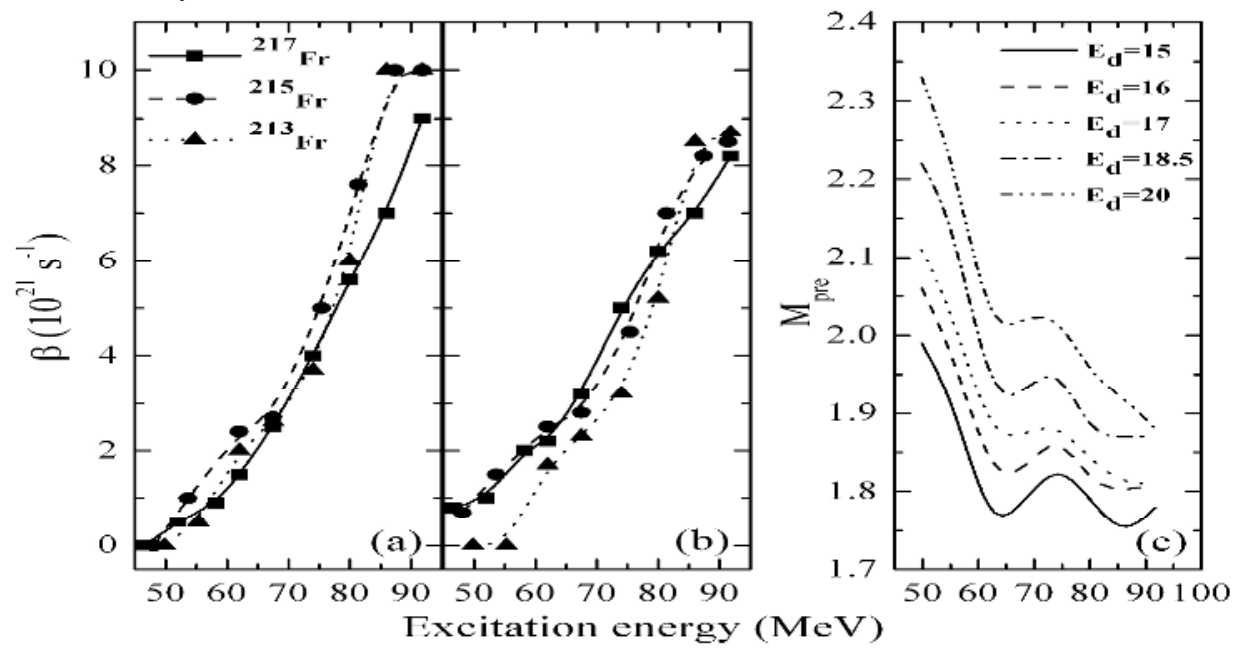

Figure 2: Comparison of the dissipation strength $(\beta)$ required to fit the experimental $\mathrm{M}_{\text {pre }}$ for different isotopes of Fr using experimental masses, shell corrected level density and (a) without shell correction in fission barrier (b) with shell correction in fission barrier, (c) Excitation function of $\mathbf{M}_{\text {pre }}$ for different values of the damping term $\left(\mathrm{E}_{\mathrm{d}}\right)$ using BW fission width for ${ }^{213} \mathrm{Fr}$.

We shall now present results from statistical model calculations where the binding energies of the evaporated particles are obtained from the experimental nuclear masses instead of the LDM masses as was done in the preceding calculations. Figure 2(a) shows the comparison of the dissipation strengths required to fit the experimental $\mathbf{M}_{\text {pre }}$. It is observed that the lowering of dissipation strength for shell closed $\mathrm{CN}$ as observed in Figure 1(b) has disappeared and nearly the same magnitude of dissipation strength reproduces the experimental $\mathrm{M}_{\text {pre }}$ for all the three $\mathrm{CN}$. The above observation can be qualitatively understood as follows. The neutron separation energy increases for ${ }^{213} \mathrm{Fr}$ whereas it decreases for the other two isotopes when experimental masses are used instead of the LDM masses. As a result, $\mathrm{M}_{\text {pre }}$ decreases for ${ }^{213} \mathrm{Fr}$ but increases for ${ }^{217} \mathrm{Fr}$ and ${ }^{215} \mathrm{Fr}$ for a given value of the dissipation strength in the statistical model calculation. Consequently a higher value of 
dissipation strength is required to fit the experimental $\mathrm{M}_{\text {pre }}$ for ${ }^{213} \mathrm{Fr}$ in comparison to calculations using the LDM masses, whereas lower values of dissipation are required for the other two isotopes. This causes the dissipation strengths for the three $\mathrm{CN}$ to come closer as shown in Figure 2(a).

Finally, shell corrections are incorporated in the fission barrier keeping everything else the same. Here the excitation energy dependent shell corrections have been employed in the fission barrier using the prescription suggested by Aritomo [10]. The parameterized form of the excitation energy dependent fission barrier used in the calculation is given as;

$$
V_{b}\left(E^{*}\right)=V_{L D M}-\delta W \exp \left(-E^{*} / E_{d}\right)
$$

where $V_{L D M}$ is the fission barrier from finite range rotating LDM potential, $\delta \mathrm{W}$ is the shell correction, $E^{*}$ is the excitation energy and $E_{d}$ is the shell damping term. It has been observed that $\mathrm{M}_{\text {pre }}$ is sensitive to the variation in $E_{d}$ as is shown in Figure 2(c). The value of $E_{d}$ is chosen to be 18.5 [6], the same value as is used for shell correction in the level density parameter. Shell correction increases the fission barrier for all the Fr isotopes under consideration and hence the statistical model predicted $\mathrm{M}_{\text {pre }}$ is expected to increase for a given dissipation strength. Consequently the fitted values of $\beta$ from statistical model calculations are reduced when shell correction is applied to the fission barrier (Figure 2(b)). The magnitude of shell correction $\delta \mathrm{W}$ is the largest for ${ }^{213} \mathrm{Fr}$ among the three Fr isotopes under consideration and further, the shell correction to fission barrier is larger at lower excitation energies. This causes the fitted values of $\beta$ for ${ }^{213} \mathrm{Fr}$ to become smaller than those of the other two Fr isotopes at small excitation energies (Figure 2(b)). The error or uncertainty in the fitted values of the dissipation strength $(\beta)$ arising out of the error bars in the experimental values for all the calculations are less than $\pm 10 \%$ of the respective mean values and these error bars do not affect any of the above discussions.

\section{Summary and Conclusions}

The experimentally obtained neutron multiplicities for different Francium isotopes are compared with the statistical model predictions using both Bohr-Wheeler and Kramers' fission widths, with and without inclusion of shell corrections in level density, nuclear masses and fission barrier. It is observed that the Bohr-Wheeler fission width under predicts the neutron multiplicity and an excitation energy dependent dissipation strength is required to fit the experimental data using Kramers' fission width. The inclusion of shell corrections in various parameters (level density, nuclear masses and fission barrier) changes the magnitude of dissipation to a large extent. In particular, shell correction in ground state nuclear masses, as given by the experimental masses, is crucial in giving similar dissipation strengths for all the Fr isotopes. Hence one can conclude that inclusion of shell corrections in various statistical model parameters is essential to estimate the magnitude of dissipation.

\section{References}

1. D. Hilscher and H. Rossner, Ann. Phys. Fr. 17, 471 (1992).

2. M. Thoennessen and G. F. Bertsch, Phys. Rev. Lett. 71, 4303 (1993).

3. Varinderjit Singh et al., Phys. Rev. C 86, 014609 (2012).

4. P. Frobrich and I. I. Gontchar, Phys. Rep. 292, 131 (1998).

5. A. J. Sierk, Phys. Rev. C 33, 2039 (1986).

6. A. V. Ignatyuk et al., Yad. Fiz. 21, 485 (1975), Sov. J. Nucl. Phys. 21, 255 (1975).

7. J. Sadhukhan and S. Pal, Phys. Rev. C 79, 064606 (2009).

8. Varinderjit Singh et al., Phys. Rev. C 87, 064601 (2013).

9. B. B. Back et al., Phys. Rev. C 60, 044602 (1999).

10. Y. Aritomo, Nucl. Phys. A 780, 222 (2006). 